\title{
PRODUCTS OF INFINITE-DIMENSIONAL SPACES
}

\author{
DALE M. ROHM
}

(Communicated by James E. West)

\begin{abstract}
Observations concerning the product of R. Pol's weakly infinitedimensional uncountable-dimensional compactum with various spaces are made. A proof showing that the product of a $C$-space and a compact $C$ space is again a $C$-space is given. Related questions, motivated by this result, are asked.
\end{abstract}

\section{INTRODUCTION}

By a space we mean a metric space and by the dimension of a space we mean the Lebesgue covering dimension, for example as presented in [E1]. A space is called countable-dimensional if it can be written as the union of a countable number of finite-dimensional subspaces. If these subspaces can be chosen to be closed in $X$, then $X$ is said to be strongly countable-dimensional. A space $X$ is weakly infinite-dimensional (in the sense of Alexandroff) if for every countable family of pairs of disjoint closed subsets $\left\{\left(A_{n}, B_{n}\right): n \in \mathbf{N}\right\}$ there exists a closed separator $S_{n} \subset X$ of each pair $\left(A_{n}, B_{n}\right)$ so that the $\bigcap\left\{S_{n}: n \in \mathbf{N}\right\}=\varnothing$. If a space $X$ is not weakly infinite-dimensional, then the space is said to be strongly infinite-dimensional. An excellent survey of these topics is contained in [EP].

A space $X$ has property $C$ and is said to be a $C$-space if for any given sequence of open covers $\left\{\mathscr{U}_{n}: n \in \mathbf{N}\right\}$ there exists a refinement $\mathscr{V}_{n}$ of $\mathscr{U}_{n}$, for each $n \in \mathbf{N}$, so that each $\mathscr{V}_{n}$ is a collection of pairwise disjoint open subsets of $X$; such a refinement is called a $C$-refinement, with the $\bigcup\left\{\mathscr{V}_{n}: n \in \mathbf{N}\right\}$ forming a cover of $X$. This covering property was originally defined by Haver for metric spaces and later generalized by Addis and Gresham to more general topological spaces. Every countable-dimensional space has property $C$, and every space with property $C$ is weakly infinite-dimensional $[\mathrm{H}][\mathrm{AG}]$. In particular, every finite-dimensional space has property $C$.

Received by the editors March 11, 1988 and, in revised form, May 20, 1988. Presented at the 838th meeting of the AMS in Los Angeles, California, November 1987.

1980 Mathematics Subject Classification (1985 Revision). Primary 54F45, 54B10; Secondary $57 \mathrm{~N} 20$.

Key words and phrases. Property $C$, weakly infinite-dimensional spaces, countable-dimensional spaces, product spaces. 
The following are well-known facts following directly from the definitions. More detail may be found in $[\mathrm{G}]$ and $[\mathrm{AG}]$.

Subspace Theorems. Every closed subspace of a weakly infinite-dimensional space (a C-space) is weakly infinite-dimensional (a C-space).

Sum Theorems. If a space $X=\bigcup\left\{X_{n}: n \in \mathbf{N}\right\}$, where each subspace $X_{n}$ is weakly infinite-dimensional (a C-space), then $X$ is also weakly infinitedimensional (a $C$-space).

Local Theorems. If every point of a space $X$ has a neighborhood which is weakly infinite-dimensional (a $C$-space), then $X$ is also weakly infinite-dimensional ( $a$ C-space).

It is not known whether every weakly infinite-dimensional space has property $C$; however, R. Pol has constructed a weakly infinite-dimensional compactum containing a strongly infinite-dimensional subspace. Hence, this compactum cannot be countable-dimensional [P1]. When constructed as a subspace of the Hilbert cube, Pol's compactum $P$, which is known to have property $C$, has the form $P=X \cup Z$ where $X$ is a topologically complete totally disconnected strongly infinite-dimensional subspace of the Hilbert cube with $Z=P \backslash X$ countable-dimensional. Thus, there are disjoint Bernstein sets $B_{1}$ and $B_{2}$, i.e., all compact subsets of $B_{1}$ or $B_{2}$ are countable, so that $Z=B_{1} \cup B_{2}$ [P2].

\section{EXAMPLES}

It is obvious from the subspace theorems that if $X$ and $Y$ are spaces whose product $X \times Y$ is weakly infinite-dimensional (a $C$-space), then both factors $X$ and $Y$ must also be weakly infinite-dimensional ( $C$-spaces).

The general converse to this statement is false. Indeed, using the notation given at the end of $\S 1$, it is known that $B_{1} \cup X$ and $X \cup B_{2}$ have property $C$ [EP], and thus are weakly infinite-dimensional. However, the product $\left(B_{1} \cup\right.$ $X) \times\left(X \cup B_{2}\right)$ contains $X \times X$ as a closed subspace, and so must be strongly infinite-dimensional [P2]. Neither factor could be compact, for if say $B_{1} \cup X$ were compact, then the projection to the other factor $X \cup B_{2}$ would be a closed mapping. This would make $X$ a closed strongly infinite-dimensional subspace of $X \cup B_{2}$, contradicting the weak infinite-dimensionality of $X \cup B_{2}$. This example motivates the following question which we discuss in $\S 3$ of this paper.

Question 1. When is the product of two weakly infinite-dimensional spaces ( $C$ spaces) again weakly infinite-dimensional (a $C$-space)?

It seems difficult to formulate positive product theorems for these infinitedimensional dimension theories. Addis and Gresham have given the following product theorem for property $C[\mathrm{AG}]$.

Theorem 1. Let $X$ and $Y$ be two $C$-spaces. If $Y$ is compact and has a basis $\mathscr{B}$ of open sets such that for all $B \in \mathscr{B}$ the product $X \times B d y(B)$ has property $C$, then the product $X \times Y$ also has property $C$. 
Corollary. If a space $X$ has property $C$ and $Y$ is a $\sigma$-compact strongly countable-dimensional space, then $X \times Y$ has property $C$.

Proof. Since the space $Y$ is $\sigma$-compact, $Y=\bigcup\left\{Y_{n}: n \in \mathbf{N}\right\}$ where each $Y_{n}$ is a compact. Moreover, since $Y$ is a strongly countable-dimensional space it is possible to write $Y=\bigcup\left\{Z_{m}: m \in \mathbf{N}\right\}$ where each $Z_{m}$ is a closed finitedimensional subspace of $Y$. Since $Y_{n}=\bigcup\left\{Z_{m} \cap Y_{n}: m \in \mathbf{N}\right\}$ for each $n \in \mathbf{N}$, it is seen that each compact subspace $Y_{n}$ is also strongly countable-dimensional. An inductive application of the theorem gives that each produce $X \times\left(Z_{m} \cap Y_{n}\right)$ has property $C$, and thus we can apply the sum theorem once to $X \times Y_{n}=$ $\bigcup\left\{X \times\left(Z_{m} \cap Y_{n}\right): m \in \mathbf{N}\right\}$ and again to $X \times Y=\bigcup\left\{X \times Y_{n}: n \in \mathbf{N}\right\}$ to see that $X \times Y$ has property $C$.

$Y$. Hattori has pointed out that $Y$ needs only to be a $\sigma$-compact countabledimensional space, since a compact countable-dimensional space has small transfinite inductive dimension [E2, Corollary 4.7]. A simple argument using transfinite induction will then give the result. However, E. Pol has shown that the compactness of the countable-dimensional factor cannot be dropped [P].

Theorem 2. There exists a C-space whose product with a subspace of the irrationals is strongly infinite-dimensional, and thus cannot have property $C$.

\section{Product theorems}

In this section, we consider products of $C$-spaces, and, in particular, we consider the product of Pol's compactum with various weakly infinite-dimensional spaces.

Theorems 3. The product of a $C$-space with a compact $C$-space is again a $C$ space.

Proof. Let $X$ and $Y$ be $C$-spaces with $Y$ compact. Given a sequence of open covers of the product $X \times Y$, we rewrite the sequence as a countable collection $\left\{\left\{\mathscr{U}_{m, n}: n \in \mathbf{N}\right\}: m \in \mathbf{N}\right\}$ of sequences of open covers. Moreover, we may assume that each cover $\mathscr{U}_{m, n}$ is of the form

$$
\mathscr{U}_{m, n}=\left\{A_{m, n}^{\alpha} \times B_{m, n}^{\alpha}: \alpha \in \Gamma_{m, n}\right\},
$$

where each $A_{m, n}^{\alpha}$ is open in $X$ and every $B_{m, n}^{\alpha}$ is open in $Y$.

Fix $m \in \mathbf{N}$ and let $x \in X$ be fixed but arbitrary. For each $n \in \mathbf{N}$, we use the compactness of $Y$ to choose a finite subset $\Gamma_{m, n}(x)$ from the indexing set $\Gamma_{m, n}$ so that

$$
\mathscr{B}_{m, n}(x)=\left\{B_{m, n}^{\alpha}: \alpha \in \Gamma_{m, n}(x)\right\}
$$

is a finite cover of $Y$ with $x \in A_{m, n}^{\alpha}$ for each $\alpha \in \Gamma_{m, n}(x)$. Because $Y$ has property $C$, for each $n \in \mathbf{N}$ we can choose a $C$-refinement $\mathscr{D}_{m, n}(x)$ of $\mathscr{B}_{m, n}(x)$ so that the $\bigcup\left\{\mathscr{D}_{m, n}(x): n \in \mathbf{N}\right\}$ is a cover of $Y$. 
We use the compactness of $Y$ again, this time to choose a positive integer $r_{m}(x) \in \mathbf{N}$ so that the $\bigcup\left\{\mathscr{D}_{m, n}(x): n=1, \ldots, r_{m}(x)\right\}$ is a finite subcover of $Y$, and then we set

$$
A_{m}(x)=\bigcap\left\{A_{m, n}^{\alpha}: n=1, \ldots, r_{m}(x), \alpha \in \Gamma_{m, n}(x)\right\} .
$$

Since $\Gamma_{m, n}(x)$ is a finite set, $A_{m}(x)$ is an open neighborhood of $x$ in $X$. Thus, by constructing such a $A_{m}(x)$ for each $x \in X$ and defining

$$
\mathscr{A}_{m}=\left\{A_{m}(x): x \in X\right\},
$$

we obtain an open cover of $X$

In this manner, for each $m \in \mathbf{N}$ we construct such an open cover $\mathscr{A}_{m}$ of $X$. Since $X$ has property $C$, we can choose a $C$-refinement $\mathscr{C}_{m}$ of each $\mathscr{A}_{m}$ such that the $\bigcup\left\{\mathscr{C}_{m}: m \in \mathbf{N}\right\}$ covers $X$. Since each $\mathscr{C}_{m}$ is a refinement of $\mathscr{A}_{m}$, we can choose a function $\phi_{m}: \mathscr{C}_{m} \rightarrow X$ so that for each $C \in \mathscr{C}_{m}$ we have

$$
C \subset A_{m}\left(\phi_{m}(C)\right) \text {, }
$$

and thus for any $n \in\left\{1, \ldots, r_{m}\left(\phi_{m}(C)\right)\right\}$ and $\alpha \in \Gamma_{m, n}\left(\phi_{m}(C)\right)$ we have

$$
C \subset A_{m, n}^{\alpha} \text {. }
$$

Finally, for each fixed $m, n \in \mathbf{N}$, we define

$$
\begin{aligned}
& \mathscr{V}_{m, n}=\left\{C \times D: n \in\left\{1, \ldots, r_{m}\left(\phi_{m}(C)\right)\right\}\right. \text { and } \\
& \left.\qquad D \in \mathscr{D}_{m, n}\left(\phi_{m}(C)\right) \text { for some } C \in \mathscr{C}_{m}\right\} .
\end{aligned}
$$

If $C \times D \in \mathscr{V}_{m, n}$, then $C \in \mathscr{C}_{m}$ with $D \in \mathscr{D}_{m, n}\left(\phi_{m}(C)\right)$, and thus $D \subset B_{m, n}^{\alpha}$ for some $\alpha \in \Gamma_{m, n}\left(\phi_{m}(C)\right)$. Therefore, we see that

$$
C \times D \subset A_{m, n}^{\alpha} \times B_{m, n}^{\alpha} \in \mathscr{U}_{m, n},
$$

so that $\mathscr{V}_{m, n}$ is an open refinement of $\mathscr{U}_{m, n}$. Moreover, since the elements of $\mathscr{C}_{m}$ are pairwise disjoint, and since for any fixed $C \in \mathscr{C}_{m}$ the elements of $\mathscr{D}_{m, n}\left(\phi_{m}(C)\right)$ are pairwise disjoint, we see that the elements of $\mathscr{V}_{m, n}$ are also pairwise disjoint.

To show that $X \times Y$ has property $C$, it only remains for us to show that $X \times Y$ is covered by the $\bigcup\left\{\mathscr{V}_{m, n}: m, n \in \mathbf{N}\right\}$. Let $(x, y)$ be an arbitrary point of $X \times$ $Y$. Since the $\bigcup\left\{\mathscr{C}_{m}: m \in \mathbf{N}\right\}$ covers $X$, we can find $m \in \mathbf{N}$ and $C \in \mathscr{C}_{m}$ with $x \in C$. Since $Y$ is covered by the $\bigcup\left\{\mathscr{D}_{m, n}\left(\phi_{m}(C)\right): n=1, \ldots, r_{m}\left(\phi_{m}(C)\right)\right\}$, we can find $n \in\left\{1, \ldots, r_{m}\left(\phi_{m}(C)\right)\right\}$ and $D \in \mathscr{D}_{m, n}\left(\phi_{m}(C)\right)$ so that $y \in D$. Hence,

$$
(x, y) \in C \times D \in \mathscr{V}_{m, n},
$$

which completes the proof.

Corollary. The product of Pol's compactum with any $C$-space has property $C$, and thus is weakly infinite-dimensional. 
Corollary. For every $n \in \mathbf{N}$, the $n$-fold product $P^{n}$ of $R$. Pol's compactum has property $C$.

Corollary. The product of a $\sigma$-compact (locally compact) $C$-space with a $C$-space is again a $C$-space.

This last corollary follows from the sum and local theorems for property $C$. Question 2. Let $f: X \rightarrow Y$ be an open and closed mapping between spaces $X$ and $Y$, where $Y$ is a $C$-space. If $f^{-1}(y)$ is a $C$-space for each $y \in Y$, then must $X$ also be a $C$-space?

\section{REFERENCES}

[AG] D. F. Addis and J. H. Gresham, A class of infinite-dimensional spaces. Part I: Dimension theory and Alexandroff's problem, Fund. Math. 101 (1978), 195-205.

[E1] R. Engelking, Dimension theory, North-Holland, Amsterdam, Polish Scientific Publishers, Warszaw, 1978.

[E2] __ Transfinite dimension theory, Surveys in Gen. Top., Academic Press, New York, 1978, 131-161.

[EP] R. Engelking and E. Pol, Countable-dimensional spaces: a survey, Dissert. Math. 216 (1983), $1-41$.

[G] D. J. Garity, Property $C$ and closed maps, Topol. Appl. 26 (1987), 125-130.

[H] W. E. Haver, A covering property for metric spaces, in Topology, Conf., Virginia Polytechnic Institute and State University, R. E. Diockman and P. Fletcher, eds., Lecture Notes in Math. vol. 375, Springer-Verlag, New York, 1974, 108-113.

[P] E. Pol, $A$ weakly infinite-dimensional space whose product with the irrationals is strongly infinitedimensional, Proc. Amer. Math. Soc. 98 (1986), 349-352.

[P1] R. Pol, $A$ weakly infinite-dimensional compactum which is not countable-dimensional, Proc. Amer. Math. Soc. 82 (1981), 634-636.

[P2] __ A remark on A-weakly infinite-dimensional spaces, Topology Appl. 13 (1982), 97-101.

Department of Mathematics, Vanderbilt University, Nashville, Tennessee 37235 